\title{
PLUG MARKERS TO DETECT ALIEN GENETIC MATERIAL IN BREAD WHEAT (Triticum aestivum L.) HYBRIDS DURING BREEDING
}

\section{P.A. SOKOLOV1, 2 , A.V. POLKHOVSKY1, 2, P.Yu. KROUPIN1, 2, V.S. RUBETS 2 , V.V. PYLNEV 2 , T.I. KHUPATSARIYA ${ }^{2}$, G.I. KARLOV1, 3, M.G. DIVASHUK 1,2}

\begin{abstract}
${ }^{1}$ K.A. Timiryazev Russian State Agrarian University-Moscow Agrarian Academy, Center for Molecular Biotechnology, 49, ul. Timiryazevskaya, Moscow, 127550 Russia, e-mail divashuk@gmail.com (corresponding author);

${ }^{2}$ K.A. Timiryazev Russian State Agrarian University-Moscow Agrarian Academy, Department of Genetics, Biotechnology, Plant Breeding \& Seed Science, 49, ul. Timiryazevskaya, Moscow, 127550 Russia, e-mail genetics@timacad.ru, selection@timacad.ru, pav2395147@yandex.ru;

${ }^{3}$ All-Russian Research Institute of Agricultural Biotechnology, Federal Agency of Scientific Organizations, 42, ul. Timiryazevskaya, Moscow, 127550 Russia, e-mail karlovg@gmail.com
\end{abstract}

ORCID:

Sokolov P.A. orcid.org/0000-0002-9301-8175

Polkhovsky A.V. orcid.org/0000-0003-0746-1902

Kroupin P.Yu. orcid.org/0000-0001-6858-3941

Rubets V.S. orcid.org/0000-0003-1233-8837

The authors declare no conflict of interests

Acknowledgements:

Supported by the grant from Russian Science Foundation, project № 16-16-00097 of January 27, 2016

Received October 11, 2016

\section{Abstract}

Molecular markers make it possible to monitor the directional introgression of alien genetic material to the genome of wheat. Among the diverse molecular markers, the PLUG (PCR-based landmark unique gene) markers have the greatest advantage for analyzing distant hybrids due to the ability to amplify polymorphism fragments on closely related species and to detect simultaneously three genomes within one homoeological group in one PCR. A collection of 41 entries of different breeding stages developed in Russian State Agrarian University as a result of crossing of bread wheat (Triticum aestivum L.) and wheat T. timopheevii Zhuk. was studied. All hybrids were resistant to leaf rust and powedery mildew natural infection. Using this collection of distant hybrids, we demonstrated for the first time the possibilities of PLUG markers for identifying alien introgressions in the genome of bread wheat from T. timopheevii. In order to increase the degree of polymorphism between the entries, we additionally processed the products of amplification of PLUG markers with highfrequency restriction endonucleases TaqI and HaeIII. The entries were found to carry both single and multiple introgressions. The latter entries with introgressions in more than one homeological group are of particular interest. The heterogeneity of not only the late generations of entries, but also the entries from the nursery of the preliminary cultivar estimation, which may be associated with secondary chasmogamy, is shown. As a result of the studies, 14 PLUG markers for the long and short chromosome arm of each homeological group have been selected, which can be recommended for the detection of the genetic material of $T$. timopheevii in distant hybrids of $T$. timopheevii $\times T$. aestivum. The advantages of PLUG markers as a tool for monitoring the transfer of alien hereditary material into the genome of bread wheat are discussed.

Keywords: common wheat, Triticum aestivum, Triticum timopheevii, wide hybridization, molecular markers, PLUG, PCR

The predominance of bread wheat (Triticum aestivum L., A ${ }^{\mathrm{u} B D}$ ) over other wheat species is determined by ecological plasticity, resistance to low and high temperatures, excess and shortage of moisture, diseases and pests. Currently, this is the main food crop for about one third of the world's population [1, 2]. However, in connection with impoverishment of genetic resources of bread wheat, evolution of pathogens, loss of efficiency of the present disease and pest resistance genes, a problem of search for new resistance genes for transfer into the $T$. aestivum genome at distant hybridization becomes important.

As a source of such genes, relatives of bread wheat are used, in particular, 
T. timopheevii Zhuk. ( $\left.\mathrm{A}^{\mathrm{b}} \mathrm{G}\right)$. In this species, a plurality of valuable resistance genes have been found, part of which has been introduced into bread wheat, e.g. two leaf rust resistance genes ( $L r 18$ and $L r 50)$, three stem rust resistance genes ( Sr36, Sr37, Sr 40), and three mildew resistance genes (Pm6, Pm27, Pm37) [3]. It is also reported that the introduced gene $\operatorname{LrTt} 2$ can be a new allele of $\operatorname{Lr} 18$ [4].

T. timopheevii wheat, which possesses resistance to brown rust, loose and covered kernel smut [5, 6], to Swedish and Hessian fly [7, 8], can be used for further enrichment of the bread wheat genotype. Moreover, T. timopheevii has increased cold resistance, high drought resistance, excess moisture resistance, and high bread baking qualities: the protein content in the seed is 19 to $30 \%$, which is significantly higher than that in the bread wheat [9-11]. Therefore, distant hybrids obtained involving $T$. timopheevii are a promising material for bread wheat selection.

The hybrid combinations of $T$. aestivum $\times T$. timopheevii were obtained in 1994 by V.F. Kozlovskaya and M.M. Starostyonkova (Altai Research Institute of Agriculture). Using these hybrids, at P.I. Lisitsyn Selection Station (Russian State Agrarian University- Timiryazev Moscow Agricultural Academy) a sample collection has been provided involving the genetic material of T. timopheevii. These samples (populations, families, lines) are the breeding products of different completeness which were selected for yielding and resistance to mildew and brown rust at natural infectious background [12].

Molecular markers are widely used in order to reveal introgressions in the progeny of distant hybrids, which can hardly be identified phenotypically. There are different marker types (expressed sequence tag-simple sequence repeats, EST-SSR; expressed sequence tag-sequence-tagged sites, EST-STS; SSR; random amplification of polymorphic DNA, RAPD; etc.) which allow detecting alien genetic material by polymerase chain reaction (PCR) and direct identifying by marker-assisted selection - MAS. For hexaploid bread wheat, PLUG (PCRbased landmark unique gene)-PCR markers are the most convenient. These markers have been developed by G. Ishikawa et al. [13] basing on conservative orthologous genes. The primers for the PLUG markers have been selected on exon sites that flank the intron. Due to the fact that in cereals the exons are high conservative, the PLUG markers developed for bread wheat, due to high homology, can be amplified using DNA of closely related species as well. At the same time, the inner parts of the amplicon are introns in which high species variability is observed (insertions/deletions, nucleotide replacements). Therefore, the products of amplification in various species would be different in size or nucleotide composition. One can detect such differences by electrophoretic separation of the PCR products (directly or, in the second case, after pre-treatment with restriction endonucleases). The main advantage of these markers is a simultaneous identifying in the distant bread wheat hybrids both all three own sub-genomes and alien introgressions within one homeological group [14].

PLUG markers allowed identifying alien chromosome obtained from other genera, e.g. Thinopyrum elongatum [15], Th. intermedium [16], Leymus mollis [17], Th. ponticum [18], in the distant hybrids of bread wheat. However, there is not a single paper on using PLUG markers to study distant hybrids from crossing Triticum genus representatives.

For the first time, we have carried out the investigation of hybrids of two wheat species from the working collection of selected samples using molecular PLUG markers and found forms with both single and multiple introgressions (from the latter, of special interest are the samples with introgressions in more than one homeological group). Heterogeneity was shown not only for latter generations of selection lines, but also for the line from the nursery of the prelimi- 
nary cultivar estimation, which can be associated with secondary chasmogamy.

The aim of the present paper was to find genetic material of Triticum timopheevii in the distant hybrid samples using PLUG markers in the course of breeding.

Technique. The collection of $\mathrm{F}_{7}-\mathrm{F}_{10}$ distant hybrids (P.I. Lisitsyn Selection Station at Russian State Agrarian University - Timiryazev Moscow Agricultural Academy, collection is based on the hybrids ontained by V.F. Kozlovskaya and M.M. Starostyonkova in Altai Research Institute of Agriculture) comprised the lines from the combination of L-6 [ 9 (Zhnitsa $\times$ T. timopheevii K-47793) $\times$ đZhnitsa] , i.e. L-6-1, L-6-2, L-6-3, L-6-4, L-6-5, L-6-6, L-6-7, L-6-8, L-6-9, L-6-10, L-6-11, L-6-12, L-6-13, L-6-14, L-6-15, L-6-16, L-6-17, L-6-18, L-6-19, and also the lines from the combination L-25 [ 9 (Novosibirskaya $67 \times$ T. timopheevii K-47793) $\times{ }^{\top}$ Novosibirskaya 67], i.e. L-25-11, L-25-12, L-2513, L-25-14, L-25-15, L-25-16, L-25-17, L-25-18, L-25-19, L-25-20, L-25-21, L-25-22, L-25-23, L-25-24，L-25-25，L-25-26，L-25-27，L-25-28，L-25-29, L-25-30. All these lines were first selected for productivity and resistance to mildew and leaf rust under natural infection. The L-6-6, L-6-7 and L-6-10 lines were involved in further selection. The parental forms of the distant hybrids, Zhnitsa and Novosibirskaya 67 bread wheat varieties, and T. timopheevii K-47793 served as control.

The genome DNA was extracted by CTAB method from the etiolated seedlings by J.J. Doyle and J.L. Doyle procedure [19].

Basing on the literature data, 52 molecular PLUG markers were selected for PCR analysis $[10,12]$. The PCR mixture $(25 \mu \mathrm{l})$ consists of $0.5 \mu \mathrm{l}$ genomic DNA (500-1300 ng/ $\mu \mathrm{l}), 2.5 \mu \mathrm{l}$ dNTP (Thermo Fisher Scientific, USA), $2.5 \mu \mathrm{l}$ Taq-buffer with $25 \mathrm{mM} \mathrm{MgCl}_{2}$ (Silex, Moscow), $1 \mu$ l forward and reverse primers (in total; Sintol, Moscow), $0.5 \mu$ l Taq-polymerase colored $(2.5 \mathrm{u} / \mu \mathrm{l}$, 5000 UA, Silex, Moscow). Amplification protocol was as follows: initial denaturation at $94{ }^{\circ} \mathrm{C}$ for $5 \mathrm{~min} ; 35$ cycles including $94{ }^{\circ} \mathrm{C}$ for $45 \mathrm{~s} ; 55{ }^{\circ} \mathrm{C}$ for $45 \mathrm{~s}$; $72{ }^{\circ} \mathrm{C}$ for $2 \mathrm{~min}$; final elongation at $72{ }^{\circ} \mathrm{C}$ for $10 \mathrm{~min}$ (a DNA engine Tetrad 2 amplifier, Bio-Rad, USA). The PCR product was treated with restriction endonucleases, TaqI and HaeIII, for $12 \mathrm{~h}$ at 65 and $37{ }^{\circ} \mathrm{C}$, respectively, according to the manufacturer's protocol (SibEnzim-M, Russia). Amplicons and their restricts were separated by electrophoresis in $2 \%$ agarose gel with TBE buffer at $6 \mathrm{~V} / \mathrm{cm}$; $100 \mathrm{bp}$ Ladder (Fermentas, Lithuania) was a molecular weight marker.

Results. In the collection, the populations omskaya $36 \times{ }^{\lambda} \mathrm{L}-6-7$, T Trizo $\times \delta^{\lambda} \mathrm{L}-6-7$, $q$ Esther $\times{ }^{\lambda} \mathrm{L}-6-10$ are in the hybridization nursery, the popu-

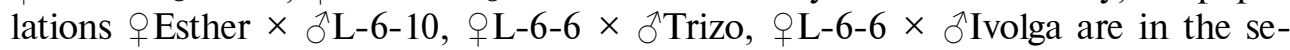

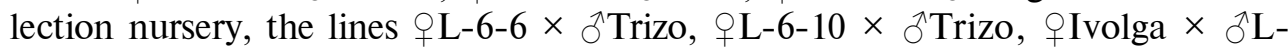
6-7, $\varnothing \mathrm{L}-6-7 \times \delta^{\top}$ Trizo are in the nursery of the preliminary cultivar estimation, and the line $9[($ Zhnitsa $\times T$. timopheevii K-47793 $) \times$ Zhnitsa $] \times \delta$ Trizo is involved in the variety testing.

Successful use of molecular markers in selection, when dealing with distant hybrids, necessitates polymorphism between the parental forms. The PLUG markers used by us were selected in such a way so for each chromosome arm of each homeological group three fragments would be amplified that are characteristic of each bread wheat sub-genome: TNAC 1009 (1S), TNAC 1010 (1S), TNAC 1026 (1L), TNAC 1102 (2S), TNAC 1178 (2S), TNAC 1233 (2S), TNAC 1021 (2L), TNAC 1118 (2L), TNAC 1204 (2L), TNAC 1248 (3S), TNAC 1300 (3S), TNAC 1263 (3L), TNAC 1383 (3L), TNAC 1408 (4AS 4BDL), TNAC 1421 (4AS 4BDL), TNAC 1428 (4AS 4BDL), TNAC 1457 (4AL 4BDS), TNAC 1464 (4AL 4BDS), TNAC 1510 (4AL 4BDS), TNAC 1663 (4AL 4BDS), TNAC 1485 (5S), TNAC 1497 (5S), TNAC 1588 (5S), TNAC 1496 (5B), TNAC 1528 
(5L), TNAC 1535 (5L), TNAC 1540 (5L), TNAC 1541 (5L), TNAC 1545 (5L), TNAC 1554 (5L), TNAC 1559 (5L), TNAC 1562 (5L), TNAC 1567 (5L), TNAC 1577 (5L), TNAC 1605 (5L), TNAC 1610 (5L), TNAC 1613 (5L), TNAC 1614 (5L), TNAC 1616 (5L), TNAC 1618 (5L), TNAC 1864 (5L), TNAC 1674 (6S), TNAC 1683 (6S), TNAC 1685 (6S), TNAC 1702 (6L), TNAC 1752 (6L), TNAC 1763 (6L), TNAC 1805 (7S), TNAC 1806 (7S), TNAC 1926 (7S), TNAC 1867 (7L), TNAC 1903 (7L), TNAC 1957 (7L).

Of these, after PCR with DNA of the parental forms (Zhnitsa and Novosibirskaya 67 bread wheat varieties, and T. timopheevii K-47793) and electrophoretic separation only those markers have been selected with which three fragments are amplified from each sub-genome of the $T$. aestivum (note that T. timopheevii and T. aestivum differ in such fragment profiles) (Fig. 1).

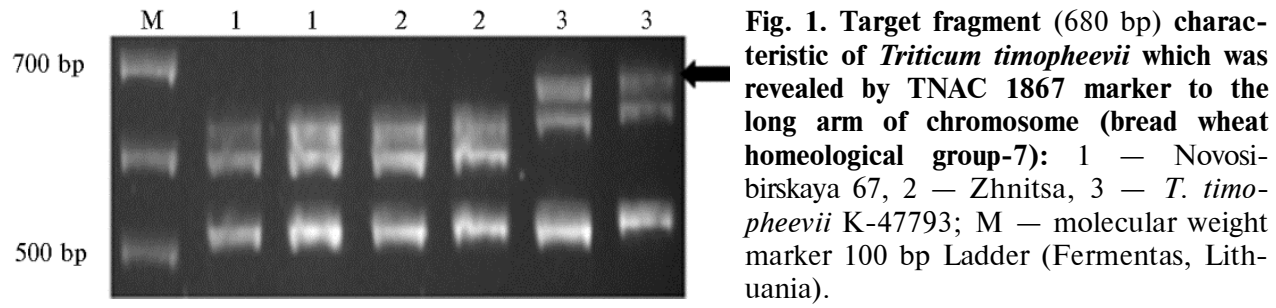

The treatment with TaqI and HaeIII, carried out for further detection of polymorphism between $T$. timopheevii and T. aestivum, has revealed 14 markers: TNAC 1026 (1L), TNAC 1010 (1S), TNAC 1021 (2L), TNAC 1178 (2S), TNAC 1383 (3L), TNAC 1248 (3S), TNAC 1464 (4AL-4BDS), TNAC 1421 (4AS-4BDL), TNAC 1535 (5L), TNAC 1485 (5S), TNAC 1752 (6L), TNAC 1674 (6S), TNAC 1867 (7L), TNAC 1806 (7S). These markers can be used for studying forms resulted from hybridization of $T$. timopheevii and $T$. aestivum, because they simultaneously detect the absence of fragments characteristic of subgenomes of bread wheat and the presence of fragments specific for T. timopheevii, indicating genomic changes in samples.

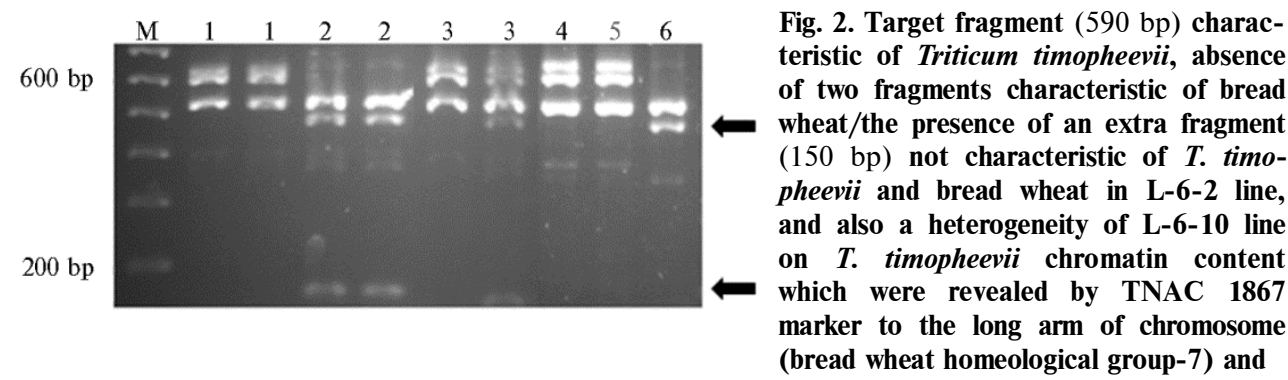

TaqI: 1 - L-6-1, 2 - L-6-2, 3 - L-6-10, 4 - Omskaya 36 variety, 5 - Esther variety, $6-T$. timopheevii K-47793; M - molecular weight marker 100 bp Ladder (Fermentas, Lithuania).

Such forms are the lines, selected from L-6 and L-25 combinations which were obtained by crossing T. timopheevii K-47793 with Zhnitsa and Novosibirskaya 67, respectively. Using the selected 14 PLUG markers, we have analyzed 19 lines from L-6 combination and 11 lines from L-25 combination and found the absence of the fragment characteristic of bread wheat and the presence of extra fragments or heterogeneity in the studied forms. It has been shown that extra fragments specific for T. timopheevii are present in the L-6-7, L-6-8, L-615, L-6-18, L-6-20 (marker TNAC 1421_TaqI), L-6-10, L-6-16, L-6-17 (TNAC 1867), L-6-2 (TNAC 1806), L-6-9, L-6-11 (TNAC 1806_TaqI) samples, and, moreover, the L-6-2 line not only contains an extra fragment of T. timopheevii DNA but also differs from the others by the absence of the fragment 
characteristic of wheat (TNAC 1867) (Fig. 2). These results indicate the introgressions from $T$. timopheevii in the genome of the said samples.

It also has been shown that in the samples L-6-6, L-6-7, L-25-26 (TNAC 1026_HaeIII); L-25-20 (TNAC 1178), L-25-21 (TNAC 1178_ TaqI); L-6-5, L-6-6, L-6-11, L-6-14, L-25-28 (TNAC 1248), L-6-3, L-25-20, L-25-21 (TNAC 1248_TaqI, TNAC 1248_HaeIII), L-25-22 (TNAC 1248_TaqI), L-25-27 (TNAC 1248 HaeIII) there is no fragment characteristic of bread wheat. In L6-16 (TNAC 1752) there was an extra fragment different to those of the bread wheat but not characteristic of T. timopheevii. In L-6-2, L-6-11, L-6-12, L-6-13, L-6-14, L-6-17 (TNAC 1752) there was no fragment characteristic of bread wheat but an extra fragment appeared not characteristic of $T$. timopheevii. The absence of the wheat fragment may indicate a presence in the corresponding locus of an alien introgression (an amplified extra fragment for such an introgression is possibly not visualized because its length corresponds to the sizes of wheat fragments). Extra fragments not characteristic of $T$. timopheevii are possibly due to polymorphism of the T. timopheevii samples. Among the studied lines, L6-2, L-6-6, L-6-7, L-6-8, L-6-10, L-6-11, L-6-12, L-6-14, L-6-16, L-6-17, L6-18, L-6-20, L-25-20, L-25-21, L-25-22, L-25-27 and L-25-28 exhibited heterogeneity which may be associated with the secondary chasmogamy.

The lines L-6-6, L-6-7 and L-6-10 were involved in producing 11 samples which selection at the moment is differently finalized. We have used 4 PLUG markers for their analysis: TNAC 1026_HaeIII (1L), TNAC 1248_TaqI (3S), TNAC 1421_TaqI (4AS-4BDL), TNAC 1867 (7L). These markers were chosen based on the fact that, according to our data, the L-6-6, L-6-7 and L-6-10 profiles differed from the bread wheat profile either in the absence of bands specific to bread wheat or in the presence of extra bands.

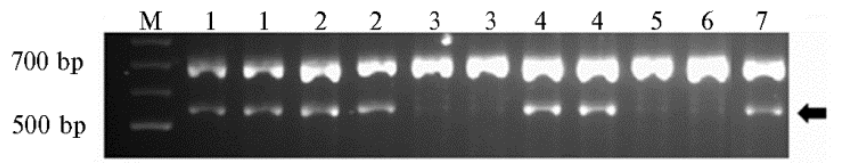

Fig. 3. Target fragment $(550 \mathrm{bp})$ characteristic of Triticum timopheevii which was revealed by TNAC 1421_TaqI marker to the long arm of chromosome of homeological group-4 for subgenome A and the long arm of chromosomes

of homeological group-4 for subgenomes $\mathbf{B}$ and $\mathbf{D}$ in bread wheat: $1-q[$ Zhnitsa $\times$ T. timopheevii $\mathrm{K}$ -

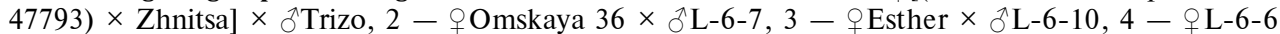
$\times$ 万Trizo, 5 - Omskaya 36, 6 - Esther, 7 - T. timopheevii M-47793; M - molecular weight marker 100 bp Ladder (Fermentas, Lithuania).

Using TNAC 1421_TaqI marker, the extra fragment of T. timopheevii type was found in the populations from the hybridization nursery 9 Omskaya 36 $\times \delta^{\wedge} \mathrm{L}-6-7$ and $\circ$ Trizo $\times{ }^{\wedge} \mathrm{L}-6-7$, in the population from the selection nursery L-6-6 $\times$ Trizo, in the line from the nursery of the preliminary cultivar estimation + Ivolga $\times \delta$ L-6-7, and also in the line involved in variety testing $9[($ Zhnitsa $\times$ T. timopheevii K-47793) $\times$ Zhnitsa $\times$ Trizo, which indicates an introgression from T. timopheevii in these samples (Fig. 3, Table). By TNAC 1248 TaqI marker, the fragment characteristic of bread wheat was not found in the populations from the hybridization nursery $\circ$ Omskaya $36 \times \delta^{\lambda} \mathrm{L}-6-7$ and $\circ$ Trizo $\times \delta^{\lambda} \mathrm{L}-6-7$, and in the lines from the nursery of the preliminary cultivar estimation 9 L-6$6 \times \delta^{7}$ Trizo and $\$$ Ivolga $\times{ }^{1} \mathrm{~L}-6-7$, which also confirms the presence of the T. timopheevii genetic material in the listed samples (see Table).

Molecular PLUG marking is the most convenient method in plant selection due to ability of simultaneous identification of the presence or absence of introduced genetic material in several subgenomes within a homeological group in polyploidy species. Using PLUG markers for studying the distant hybrids of bread wheat and T. timopheevii at sequential steps of selection, we have obtained 
data about the presence of an alien genetic material in a number of studied forms and also about the heterogeneity of some samples.

Characterization of Triticum aestivum and T. timopheevii distant hybrids' progeny at sequential steps of selection using two PLUG markers

\begin{tabular}{|c|c|c|}
\hline Sample & Selection step & PCR amplification profile \\
\hline \multicolumn{3}{|c|}{ TNAC 1248 (3S)_TaqI } \\
\hline †Omskaya $36 \times ð$ L-6-7 & Hybridization nursery & Absence of fragment characteristic of bread wheat \\
\hline Trizo $\times{ }^{\lambda} \mathrm{L}-6-7$ & Hybridization nursery & Absence of fragment characteristic of bread wheat \\
\hline + L-6-6 $\times$ Trizo & Selection nursery & Absence of fragment characteristic of bread wheat \\
\hline Ivolga $\times \gg$ L-6-7 & \multicolumn{2}{|c|}{$\begin{array}{l}\text { Preliminary cultivar estimation Absence of fragment characteristic of bread wheat } \\
\text { TNAC } 1421 \text { (4AS-4BDL)_TaqI }\end{array}$} \\
\hline †Omskaya $36 \times \precsim$ L-6-7 & Hybridization nursery & Extra fragment characteristic of T. timopheevii \\
\hline qTrizo $\times \delta^{\lambda} \mathrm{L}-6-7$ & Hybridization nursery & Extra fragment characteristic of $T$. timopheevii \\
\hline L-6-6 $\times$ OTrizo & Selection nursery & Extra fragment characteristic of $T$. timopheevii \\
\hline †Ivolga $\times \quad$ L-6-7 & Preliminary cultivar estimation & Extra fragment characteristic of $T$. timopheevii \\
\hline $\begin{array}{l}\text { I[(Zhnitsa } \times \text { T. timopheevii } \\
\text { K-47793 }) \times \text { Zhnitsa } \times 3 \text { Trizo }\end{array}$ & Variety testing & Extra fragment characteristic of T. timopheevii \\
\hline
\end{tabular}

Specific PLUG markers we used allowed three types of PCR product profiles. The first type matched that of bread wheat, which indicated the absence of introgressions. The second type was characterized by the absence of wheatspecific product. This may indicate a loss of certain chromosome site of bread wheat as a result of substitution by an alien fragment, which was not identified visually (possibly due to size matching with the wheat fragment). For the third type, the absence of the wheat fragment and the presence of the extra fragment of amplified alien introgression were characteristic.

In the earlier studies, the PLUG markers were used in wheat for identification chromatin of distant genetic species Thinopyrum elongatum [15], Th. intermedium [16], Leymus mollis [17], Th. ponticum [18]. We have shown for the first time a possibility to obtain distinctive profiles in such genetically close species as bread wheat and T. timopheevii.

Additional amplified fragments found in the studied samples and not matching to those present in the profiles of bread wheat could be associated with stability to brown rust and mildew. The PLUG markers selected by us and allowing for amplifying the specific fragments can be used for assessment of segregating populations in order to find an alien introgression associated with plant resistance. Earlier, we demonstrated the efficiency of such an approach using PLUG markers in studying 6Ai\#2(6D) substitution which determines resistance to leaf rust in the Tulaykovskaya 10 bread wheat variety [16].

We have found forms both with single and multiple introgressions. The samples with multiple introgressions in several homeological groups are of special interest. Thus, L-6-6 has introgressions in homeological groups 1 and 3, L6-7 - in homeological groups 1 and 4, L-25-20 and L-25-21 - in homeological groups 2 and 3, L-6-11 - in homeological groups 3, 6 and 7, L-6-14 - in homeological groups 3 and 6, L-6-2, L-6-16 and L-6-17 - in homeological groups 6 and 7 . The markers we chose, when using the collection of nullitetrasomics as control, allow to locate single and multiple introgressions more precisely. Taking into account that PLUG markers are high conservative, one can thus expect that extra DNA fragments in the profiles testify to alien genetic material introgressed exactly from the homeological group of $T$. timopheevii to which the corresponding PLUG marker was developed.

Moreover, by PLUG markers, the heterogeneity could be studied, and the most stable lines not segregating by markers could be revealed. In particular, we showed the heterogeneity not only in the latter generations of lines selected from the L-6 and L-25 crossing combinations, but even in the line from the nursery of preliminary cultivar estimation, + L-6- $6 \times$ ○Trizo that can be associated with secondary chasmogamy [20]. 
Thus, PLUG marking facilitates monitoring and a directed introgression of alien genetic material into the bread wheat genotypes. This is especially helpful for Triticum timopheevii, involving of which in wheat breeding is as valuable as complicated. As a whole, PLUG markers are helpful when working with distant hybrids as they are capable of identifying alien introgressions even in the absence of information about donor species, because these markers allow to conclude about the introgressions by comparing PCR product profiles in the hybrid form and in bread wheat.

\section{REFERENCES}

1. Goncharov N.P., Kondratenko E.Ya. Vestnik VOGiS, 2008, 12(1/2): 159-179 (in Russ.).

2. Zernovye kul'tury (vyrashchivanie, uborka, dorabotka i ispol'zovanie) [Grain crops: growing, harvesting, processing and usage]. D. Shpaar (ed.). Moscow, 2016 (in Russ.).

3. McIntosh R.A., Yamazaki Y., Dubcovsky J., Rogers J., Morris C., Some r s D.J., A p ples R., D e vos K.M. Catalogue of Gene Symbols for Wheat. Proc. 12 th Int. Wheat Genetics Symposium (8-13 September 2013, Yokohama, Japan). Available https://wheat.pw.usda.gov/GG2/Triticum/wgc/2013/GeneCatalogueIntroduction.pdf. No date.

4. Le onova I.N., Budashkina E.B., Flath K., Weidner A., Börner A., Röde r M.S. Microsatellite mapping of a leaf rust resistance gene transferred to common wheat from Triticum timopheevii. Cereal Res. Commun., 2010, 38: 212-220 (doi: 10.1556/CRC.38.2010.2.7).

5. M a rke lov a T.S. Zashchita i karantin rastenii, 2011, 1: 21-23 (in Russ.).

6. Z huk ovski i P.M. Izbrannye Trudy [Selected works]. V.F. Dorofeev (ed.). Leningrad, 1985 (in Russ.).

7. D o r o f e e v V.F. Pshenitsy mira [Wheats]. Leningrad, 1987 (in Russ.).

8. Brown-Guedira G.L., Gill B.S., Bockus W.W., Cox T.S., Hatchett J.H., Le ath S., Peters on C.J., Thom as J.B., $Z$ we r P.K. Evaluation of a collection of wild Timopheevii wheat for resistance to disease and arthropod pests. Plant Dis., 1996, 80: 928-933.

9. Ti mo nova E.M., L e o nova I.N., B e 1 a n I.A., Ross e eva L.P., S a lina E.A. Vavilovskii zhurnal genetiki i selektsii, 2012, 16: 142-159 (in Russ.).

10. Chastnaya selektsiya polevykh kul'tur [Field crops: particular aspects of breeding]. V.V. Pyl'nev (ed.). St. Petersburg, 2016 (in Russ.).

11. Obukhova L.V., Budashkina E.B., Ermakova M.F., Kalinina N.P., $\mathrm{S} \mathrm{h} \mathrm{u} \mathrm{m} \mathrm{n} \mathrm{y} \mathrm{i} \mathrm{V.K.} \mathrm{Grain} \mathrm{and} \mathrm{flour} \mathrm{quality} \mathrm{in} \mathrm{introgressive} \mathrm{lines} \mathrm{of} \mathrm{spring} \mathrm{soft} \mathrm{wheat} \mathrm{carring} \mathrm{leaf} \mathrm{rust}$ resistance genes from Triticum timopheevii Zhuk. Agricultural Biology, 2008, 5: 38-42 (in Russ.).

12. $\mathrm{Ru} \mathrm{b}$ e ts V.S. Biologicheskie osobennosti tritikale kak osnova sovershenstvovaniya selektsionnogo protsessa. Doktorskaya dissertatsiya [Biological features of Triticale as a tool to improve breeding technologies. DSc Thesis]. Moscow, 2016 (in Russ.).

13. Ishikawa G., Yonemaru J., Saito M., Nakamura T. PCR-based landmark unique gene (PLUG) markers effectively assign homoeologous wheat genes to A, B and D genomes. BMC Genomics, 2007, 8(1): 135 (doi: 10.1186/1471-2164-8-135).

14. Ishikawa G., Nakamura T., Ashida T., Saito M., Nasuda S., Endo T.R., $\mathrm{Wu}$ J., Mats umoto T. Localization of anchor loci representing five hundred annotated rice genes to wheat chromosomes using PLUG markers. Theor. Appl. Genet., 2009, 118(3): 499514 (doi: 10.1007/s00122-008-0916-y).

15. H u L.-J., Li u C., Z eng Z.-X., Li G.-R., S o ng X.-J., Y ang Z.-J. Genomic rearrangement between wheat and Thinopyrum elongatum revealed by mapped functional molecular markers. Genes Genom., 2012, 34(1): 67-75 (doi: 10.1007/s13258-011-0153-7).

16. Salina E.A., Adonina I.G., Badaeva E.D., Kroupin P.Yu., Stasyuk A.I., Le onova I.N., Shishkina A.A., Divashuk M.G., Starikova E.V., Khuat T.M.L., S yu k ov V.V., K a rlov G.I. A Thinopyrum intermedium chromosome in bread wheat cultivars as a source of genes conferring resistance to fungal diseases. Euphytica, 2015, 204: 91-101 (doi: 10.1007/s10681-014-1344-5).

17. Yang X., Wang C., Li X., Chen C., Tian Z., Wang Y., Ji W. Development and molecular cytogenetic identification of a novel wheat-Leymus mollis Lm\#7Ns (7D) disomic substitution line with stripe rust resistance. PLoS ONE, 2015, 10(10): e0140227 (doi: 10.1371/journal.pone.0140227).

18. Li X.J., $\mathrm{Hu}$ X.G., $\mathrm{Hu}$ T.Z., Li G., Ru Z.G., $\mathrm{Z}$ h a $\mathrm{ng}$ L.L., La ng Y.M. Identification of a novel wheat-Thinopyrum ponticum addition line revealed with cytology, SSR, EST-SSR, EST-STS and PLUG markers. Cereal Res. Commun., 2015, 43(4): 544-553 (doi: 10.1556/0806.43.2015.021).

19. D o yle J.J., D o yle J.L. A rapid DNA isolation procedure for small quantities of fresh leaf tissue. Phytochemistry Bulletin, 1987, 19: 11-15.

20. Rubet s V.S., Pyl'ne v V.V., Yalto nskay a M.V., D ivashuk M.G., Koro$\mathrm{t}$ a e v a A.A. Izvestiya TSKhA, 2012, 6: 62-69 (in Russ.). 